\title{
Capítulo I \\ Cómo comprender la guerra y por qué se requieren dimensiones para llevarla a cabo
}

Ya que la primera pregunta que compone este subtítulo se refiere

I a cuestionarse sobre qué es la guerra, es importante que el lector, más allá de esperar un sinnúmero de definiciones sobre lo que se puede entender sobre este fenómeno, sepa que encontrará elementos teóricos y conceptuales que contribuyan a dilucidar si la ciberguerra se conecta con los aspectos que normalmente se reconocen en un contexto bélico. También es importante afirmar que la guerra y otros fenómenos, como la religión, la movilización, las organizaciones sociales, han hecho parte de la evolución del ser humano, por tanto, ayudan a entender su comportamiento y su desarrollo hasta llegar a lo que es hoy.

\section{Caracterización de la guerra}

A continuación, la intención es entender las causas y las características principales de la guerra, en busca de comprenderla para lograr asociarla con el concepto de ciberguerra como acontecimiento político y social. En la actualidad, contando con tantas obras, estudios y análisis sobre la guerra, se ha podido determinar que esta ha presentado diversas causas a lo largo de la historia. A partir de la visión más primitiva del realismo político, se entiende que la causa fundamental 
primaria de la guerra ha sido la supervivencia del ser humano. Por supuesto, esta noción de supervivencia no es entendida como una lucha o una empresa por que la humanidad sobreviva como especie, sino que, gracias a la racionalidad, característica particular del hombre, esta emerge de unidades políticas conformadas por grupos sociales específicos, que buscan alcanzar intereses particulares. A lo largo del tiempo, estas unidades han transformado o evolucionado sus características.

Se tiende a utilizar diferentes rótulos para referirse a estas unidades: inicialmente, pueblos, tribus, clanes, para ir evolucionando a otras formas que son conocidas y que actualmente se denominan ciudades, Estados, imperios; y, finalizando esa etapa, los Estados monárquicos absolutistas. Siguiendo a Molina (2010), las causas de las guerras entre estas unidades se pueden encontrar en elementos como la ambición de conquista, los propósitos de colonización, de ubicación de puertos comerciales, de disputas religiosas o de incremento de los medios de subsistencia y del espacio vital de los pueblos. Pasados los siglos, cuando los pueblos colonizados llegaron hasta el punto de inflexión moral frente a las injusticias de los imperios, el objeto de la guerra fue lograr la independencia nacional — lo anterior se menciona para interés de un acápite final de este libro-. También podría señalarse el mismo objeto dentro de las guerras civiles.

Molina (2010) permite comprender de manera práctica cuáles han sido las causas de la guerra a lo largo de la historia, presentando lo que Carl von Clausewitz explicaría como el objetivo político de la guerra. No obstante, a los ojos de este mismo filósofo prusiano de la guerra, es preciso entender que también, cuando se originan escenarios de combate entre dos actores políticos, se debe tener en cuenta que el objetivo político pasa a un segundo plano y lo importante durante la coyuntura es obtener o alcanzar el objetivo militar. Por esto, y de una manera más cercana al estilo de Clausewitz, se podría aceptar que la guerra es una combinación de muchos compromisos distintos. Tal combinación puede o no ser razonable, y el éxito depende mucho de esto. Sin embargo, el compromiso en sí es lo más importante, ya que solo una combinación de compromisos exitosos puede conducir a buenos resultados: "Lo más importante 
en la guerra será siempre el arte de derrotar al oponente en combate"1 (Clausewitz, 1942, p. 4).

Reconociendo que derrotar al oponente en combate nunca significó para Clausewitz la aniquilación de un ejército o un pueblo, se podría afirmar que la guerra, por más violenta que sea, nunca ha tenido como objetivo destruir al ser humano, sino que, a través de la ejecución de actos de violencia que terminan afectando o asesinando al contrincante, se logra que la maquinaria de guerra opuesta cese su acción bélica. Por esto, es una cualidad esencial de quienes están en comando durante la guerra determinar cuál es el objetivo y que, en el momento de atacarlo, el enemigo quede sin energía para seguir luchando en el campo de batalla. No es fortuito así que para Clausewitz (1942) las hostilidades deban ir dirigidas a conquistar y destruir el poder armado del enemigo y tomar posesión de sus recursos materiales y demás fuentes de fuerza.

Para el caso concreto de los Estados nación, y por ende se habla de características modernas y westfalianas de ordenamiento político, todos los tipos de guerra (aun cuando habían obedecido a modalidades bélicas diferentes, dadas sus diferencias en objetivos, los tipos de ejércitos que empleaban, la técnica militar y la economía de guerra) compartieron rasgos comunes, ya que son catalogadas como formas de violencia organizada por Estados que recurren a ellas como mecanismo para ejercer control y legitimidad en espacios territoriales, establecer una demarcación entre lo interno y lo externo, mantener el orden interno, o bien proporcionar a los tomadores de decisiones capacidades disuasivas o de respuesta frente a la amenaza de un actor violento del sistema internacional (Fazio, 2003).

Por supuesto, y siguiendo a Fazio (2003), se trató de un momento en el que cobró validez intentar reproducir una clara diferenciación entre la guerra y los otros tipos de conflictos y violencias. Este compromiso "demandó que el Estado actuara como un agente organizador de la guerra; los ejércitos, aun cuando fuesen guerras civiles, se ordenaban jerárquicamente y la economía de guerra se estructuraba desde un punto de vista estatal” (p. 43).

1 Traducción del autor. 
Por esto, el elemento sobre el cual se concentró la guerra en la modernidad se conceptualizó gracias a Clausewitz como centro de gravedad. En primer lugar, el centro de gravedad de Clausewitz es un "punto focal", no es una fuerza, ni una fuente de una ni una debilidad per se. Por otra parte, se encuentra exclusivamente donde existe un nivel de conectividad tal entre las distintas partes del enemigo que se forma un sistema o estructura que actúa con un grado sustancial de unidad, como un cuerpo físico. En tercer lugar, un centro de gravedad ejerce cierta fuerza centrípeta que tiende a mantener un sistema o estructura entera juntos; así, un golpe en el centro de gravedad lanza a un enemigo fuera del punto de equilibrio, incluso, puede causar que el sistema entero se derrumbe. Cuarto, usar el concepto requiere ver al enemigo holísticamente (Echevarría, 2003).

Diferentes tipos de equipos e instrumentos se han introducido y utilizado en las guerras y han conformado diferentes tipos de guerra. La guerra convencional expresa la reducción de la capacidad militar de un oponente a través de la batalla abierta, la guerra no convencional es lograr la victoria militar a través de la aceptación, la capitulación o el apoyo secreto a un lado de un conflicto existente $^{2}$. (Bazyan, 2012, p. 2)

Para interés de un acápite final del presente libro, podría señalarse que, dentro de las guerras civiles que han enfrentado a naciones a partir de antagonismo en las concepciones políticas, económicas, religiosas y étnicas de los componentes de la sociedad, estas también deberían considerarse como causa de las conflagraciones bélicas (Molina, 2010).

Conforme a lo que establece Fabricio, se pueden enumerar tres cualidades que deben estar presentes para hablar de guerra: un ambiente donde se pueda llevar a cabo, tecnologías militares para su desarrollo y la doctrina, entendida como "un conjunto de técnicas, estrategias, tácticas y prácticas que constituyen un enfrentamiento bélico y propone los pasos por seguir para ganar una guerra” (s. f.).

2 Traducción del autor. 


\section{El entorno}

La guerra y el territorio han presentado una estrecha relación a lo largo de la historia. Con el surgimiento de la organización política, en el momento donde se evolucionó de jefaturas y pequeñas poblaciones a la figura del Estado nación, la principal razón de los enfrentamientos y la guerra como fenómeno era la clara necesidad de imposición como autoridad soberana por parte de quien aspiraba a convertirse en gobernante. Con el levantamiento de las primeras ciudades-Estado, la causa de la guerra fue la conquista del territorio de los pueblos vecinos (Molina, 2010, p. 113).

Respecto del primer componente, el ambiente, Sun Tzu planteaba siglos atrás que el terreno implica las distancias y hace referencia a dónde es fácil o difícil desplazarse, y si es campo abierto o lugares estrechos, puesto que influye en las posibilidades de supervivencia (Maggio, 2013, p. 82; Tzu, 1999, p. 3; Tzu, Von Clausewitz y Musashi, 2016, p. 13).

Por su parte, Droznes (2005) describe el ambiente por aquello que deben emplearlo como escenario de combate a partir de los tipos de superficie. Se pueden distinguir seis tipos de superficie, a saber: terreno accesible, terreno difícil, terreno neutro, de pasos estrechos, alturas abruptas y posiciones que se encuentran a gran distancia del enemigo. Para el autor, la formación natural del suelo es el mejor aliado del soldado.

La guerra puede ser clasificada por el ambiente o el área en la cual está ocurriendo. Esto significa que dos tipos de guerra, convencionales y no convencionales, afectan un área de acuerdo con las estrategias y la región donde necesitan actuar, y también puede ocurrir lo contrario: que los tipos de ambiente cambien el tipo de guerra. Muchos teóricos han intentado catalogar la forma de hacer la guerra conforme a los entornos donde se iba a llevar a cabo. Bazyan (2012) las cataloga entre guerra de la selva, guerra ártica, guerra de la montaña y guerra urbana. Ejemplos de guerra no convencional incluyen la guerra psicológica, la guerra biológica, la guerra electrónica y la guerra nuclear (p. 3). 


\section{La tecnología}

Hablando en términos complementarios de tecnología, Martí (2006) describe la tecnología militar como aquella parte del desarrollo tecnológico que está enfocado en la aplicación a cualquier aspecto relacionado con la seguridad, la defensa y la eficacia en el enfrentamiento. Desde esta línea de pensamiento, las invenciones que allí se producen se enfocan en resolver los problemas fundamentales que se le presenten, entre ellos: la capacidad de destrucción, la protección propia, la movilidad eficiente, el mando, el control, las comunicaciones y el apoyo logístico.

En otro sentido, la relación de la guerra con la tecnología también se ha derivado de la capacidad de evolución de la industria bélica y lo que esta, al final del camino, le pueda ofrecer al soldado para desempeñar mejor su labor en el campo de batalla. Black, por ejemplo, permite comprender este aspecto cuando estudia, desde la perspectiva europea, lo que significó para los ejércitos propios de los siglos XV al XVII dejar atrás las ballestas para emplear armas de fuego como los arcabuces, las pistolas de la época, o bien armas largas como el mosquete o los pedreñales (2009, p. 98). Esta idea es reforzada con superioridad cuando se hace referencia a la gran obra de Martin van Creveld, Technology and war.

La evolución de las armas en la guerra, así como ha determinado la consecución de más poder para los Estados, también ha demandado responsabilidades en sus instituciones castrenses. Se trata de comprender que la transformación de la guerra no simplemente implica concebir o desarrollar nuevas tecnologías y arquetipos, sino también establecer las instrucciones que los militares deben seguir para poder aprovechar las capacidades de los armamentos en el momento de emplearlos. En palabras de Cooper (1997), las nuevas capacidades no solo deben desarrollarse físicamente y demostrar su superioridad, sino que la implementación exitosa de las innovaciones requiere un proceso complejo de integración en la estructura de las fuerzas militares y en los condicionamientos operacionales. La adopción de la innovación exige más que solo la capacidad de equipar una fuerza o un servicio militar con armas innovadoras; las organizaciones, los patrones 
operativos y los procesos de toma de decisión también deben ser modificados para implementar la innovación como un elemento integral del carácter distintivo del servicio (p. 119).

La era de la máquina, como denomina Davis (1997) al momento de industrialización de las guerras desde finales del siglo xIx y hasta las guerras mundiales, fue una época dirigida a la concatenación de habilidades que promovieron el aprovechamiento del potencial militar de una manera cada vez más orquestada, jerarquizada e institucionalizada, pero, a su vez, descentralizada, como lo demostró la Wehrmacht (Fuerzas Armadas de la Alemania nazi) en la Segunda Guerra Mundial. Fue un momento de aprovechamiento de los vastos recursos de los Estados para dar vida a los ejércitos y a la guerra en masas (p. 82).

Otra realidad alcanzada entre la tecnología y la guerra fue la cultura de la integración de artefactos preexistentes con nuevos adelantos en armamentos. Por ejemplo, hacer de los trenes de pasajeros máquinas de combate al emplazarlos como cañones y blindajes, instalar radios de comunicación en los diferentes medios de transporte, dotar de ametralladoras de alto calibre a los aviones de caza. Esta era culminó en la Segunda Guerra Mundial con la innovadora aplicación de la mecanización, la aviación y la tecnología de las comunicaciones al uso militar, lo que permitió al ejército alemán reintroducir la movilidad estratégica y operativa ${ }^{3}$. (Davis, 1997, p. 82)

Esto recuerda el panorama descrito por William S. Lind en Fifth generation warfare. A partir de los adelantos científicos que emergieron de la Guerra Fría, se dio un incremento exponencial frente a la cultura de la integración tecnológica señalada. En este momento, el desarrollo tecnológico no se trata específicamente de armas y vehículos, por ejemplo, sino de la información y de cualquier cosa que la industria militar quisiera integrar con esta. Gran parte de la maquinaria, los dispositivos, las armas, los vehículos e, incluso, los mismos soldados que fabricaron y alimentaron las dos superpotencias durante

3 Traducción del autor. 
la Guerra Fría demandaron grandes cantidades de información para ejecutar sus funciones, tomar decisiones, emprender misiones y realizar operaciones, campañas o guerras. El aumento de la cantidad de información que debe ser digerida para que los propósitos bélicos se lleven a cabo se convirtió en una tarea tan compleja que obligó a la adaptación casi inmediata de la informatización y automatización de los procesos de recopilación, análisis y distribución de información, lo que permitió a comandantes y soldados responder a las velocidades adquiridas por el ejercicio de la guerra (Davis, 1997, p. 82).

Para comprender la evolución de la variable tecnológica, solo hay que imaginar lo que hubiera sido lanzar un misil nuclear balístico desde los Estados Unidos sin la capacidad científica y tecnológica para integrar estos procesadores de información que guiaran su trayectoria de vuelo hasta la antigua Unión Soviética, así como la altura a la cual activar el mecanismo de detonación al llegar a su objetivo; todo esto debía estar informatizado y automatizado. Como se expondrá a lo largo de este libro, la informatización de la guerra, y muchos asuntos importantes para los Estados y sus sociedades, abrieron la puerta a la ciberguerra, la cual contiene las mismas características de la guerra y, por tanto, afecta todos los escenarios en los que se desarrolla la vida humana.

Al recapitular, es posible decir que existen dos variables que han ayudado a determinar o dar ciertas connotaciones especiales a la guerra. Un primer elemento determinado como terreno y, de este, la descripción frente al hecho del lugar en que se va a combatir. Y un segundo, el elemento tecnológico, el cual, más allá de ofrecer categorías, termina siendo uno de los fenómenos más decisivos en el momento de abordar las grandes transformaciones de la guerra. Ahora bien, aunque ambos elementos puedan encontrarse en vías diferentes, tanto la definición de las condiciones en las que se va a pelear, así como el equipo diseñado y empleado para el enfrentamiento, demandan al unísono otra forma más de transformación: el cambio en la doctrina.

\section{La doctrina}

Como ya se había anunciado, los cambios que llevaron a la ciberguerra no solo se concentraron en las preguntas sobre en dónde se lucha la 
guerra y con qué se puede luchar en la guerra, sino que también debe incluirse el cómo se debe luchar. La estrategia significa la combinación de compromisos individuales para alcanzar el objetivo de la campaña o la guerra. Retomando a Clausewitz, "es cierto que, si sabemos cómo luchar y cómo ganar, muy poco conocimiento extra es necesario en la guerra para combinar resultados afortunados. Es solo una cuestión de juicio y experiencia"4 (Clausewitz, 1942, p. 17).

Es importante reconocer que este cambio en las técnicas para llevar a cabo la guerra se puede entender como una revolución, pero no simplemente siendo una condición existencial; por tanto, no es creada simplemente por la aparición de nuevas capacidades tecnológicas. Sin el reconocimiento y la explotación, donde ambos requieren una acción positiva, no puede haber revolución. "Crear una revolución es, por tanto, más que empujar los límites de la tecnología militar; es un proceso activo que requiere una adaptación efectiva por parte de los individuos y las organizaciones para que exista una explotación exitosa"5 (Davis, 1997, p. 80).

Este conocimiento ha tenido que ser transmitido a la estructura militar para que cada miembro o unidad que la compone tenga los parámetros de comportamiento que deba asumir a fin de dar respuesta a las diferentes situaciones que se pueden presentar en el ámbito castrense, o bien para la guerra, o bien para actividades de contención o rutina. Por esto, se requieren planes para preparar todos los instrumentos nacionales de poder para la guerra $o$ conflicto, una guía práctica para preparar las Fuerzas Armadas y el liderazgo de estas para alcanzar objetivos estratégicos. El nivel doctrinal se refiere al empleo de fuerzas militares en un teatro de guerra o un teatro de operaciones para obtener una ventaja sobre el enemigo y así lograr objetivos estratégicos a través del diseño, la organización y la conducción de campañas y operaciones importantes. En la guerra, una campaña implica el empleo de las fuerzas

\footnotetext{
4 Traducción del autor.

5 Traducción del autor.
} 
militares en una serie de operaciones militares relacionadas a fin de lograr un objetivo común en un tiempo y un espacio dados. Los comandantes deben diseñar, orquestar y coordinar operaciones y explotar eventos tácticos con el propósito de apoyar los objetivos generales de la campaña ${ }^{6}$. (USAF College of Aerospace Doctrine, Research and Education, 1997, pp. 1-2)

De este nivel estratégico mencionado, se desprenden los niveles operacionales y tácticos (USAF College of Aerospace Doctrine, Research and Education, 1997, p. 2). El nivel táctico traduce el poder potencial real de combate que puede presentar un determinado Estado a través de sus fuerzas en las batallas y compromisos a través de decisiones y acciones que crean ventajas cuando están en contacto o en las proximidades del enemigo. Las tácticas se ocupan de los detalles de los juicios y son extremadamente sensibles al entorno cambiante del campo de batalla.

Por otra parte, y relacionados de forma directa con la doctrina o estrategia para llevar a cabo la guerra, los principios de esta son aquellos que expresan las reglas del pensamiento militar y las acciones militares que sirven como base permanente para la doctrina de combate. Su importancia relativa variará de un evento a otro. La lista de principios es una herramienta metodológica que difiere de ejército a ejército y de época en época. Mientas los principios se mantienen iguales, la lista se transforma según el tiempo y el lugar y la aplicación siempre depende del contexto (Mallick, 2009, p. 2). Los principios de la guerra han evolucionado durante un largo periodo. La evolución se puede clasificar en tres etapas: la primera, antes de la era de la guerra napoleónica; un segundo momento desde la era napoleónica hasta el final de la Segunda Guerra Mundial; y el último espacio temporal de la pos-Segunda Guerra Mundial (p. 3).

Modernizar los principios tradicionales de la guerra para los conflictos del siglo xxI no hace irrelevantes las versiones anteriores. Por el contrario, la intención de modernizar los principios de la guerra es capturar el espíritu de los existentes; por ejemplo, la información y la

6 Traducción del autor. 
adaptabilidad. En resumen, el objetivo no es el reemplazo al por mayor de los principios tradicionales en sí, sino una renovación consciente del valor duradero de los antiguos. Los principios modernizados de la guerra podrían servir de guías para los líderes militares y civiles involucrados en los conflictos del siglo xxi (Dunlap, 2006, pp. 42-47). Tales principios podrían ayudar a la conducción de la guerra y ayudar a su organización, entrenamiento y equipamiento.

Dentro de este ámbito, también suele presentarse el proceso de absorción y adaptación a los nuevos instrumentos para hacer la guerra. Y este es un tema de suma vitalidad para la ciberguerra. El proceso de desarrollo tecnológico militar no ha tenido límites, pues siempre ha conllevado la introducción de nuevo armamento en las fuerzas militares, y se ha generado un constante conflicto entre el derecho en la guerra (ius in bellum) y las nuevas tecnologías. Muchas tecnologías, en especial las armas, se han percibido, al menos inicialmente, en desacuerdo con la ley existente.

La historia de la guerra ha sido puntuada repetidamente por las acusaciones de que ciertas armas nuevas son ilegales, por cuanto son injustas de acuerdo con los criterios prevalecientes de honor, equidad y demás, o porque su acción es más desagradable de lo que necesita ser. Cualquiera que sea la naturaleza y la fuerza de las objeciones al principio encontradas, las nuevas armas se deslizan en el uso común tan pronto como los objetores pueden adquirirlas por sí mismos, con lo cual la ley se adapta en consecuencia. Respecto de lo anterior, Liivoja (2015) explica que lo importante es que el cambio tecnológico no es un nuevo tipo de desafío para el derecho de la guerra. Lo que bien podría ser cierto, sin embargo, es que el cambio tecnológico está ocurriendo mucho más rápido ahora, con lo cual se exacerba el problema de que la ley tiende a quedarse atrás de la tecnología (p. 1173).

Los tres elementos que se han señalado como características de la guerra - entorno, tecnologías y doctrina- facilitan, hasta el momento, proseguir al elemento final de análisis. A continuación, un último paso sobre los escenarios en los que se lleva a cabo el enfrentamiento permitirá asumirlo como un tema central en la comprensión de la ciberguerra: dónde se lleva a cabo esta práctica y qué ocurrió para que existiera. 


\section{Las dimensiones de la guerra}

A partir de la existencia y validez que han presentado los poderes en la historia militar, ha sido evidente que su aparición se debió a la tendencia del hombre a extender el empleo de la fuerza a nuevos escenarios de enfrentamiento con el fin de desarrollar nuevos tipos de estrategias para ganar la guerra. Consecuentemente, en una primera instancia, el conflicto se desenvolvió en los teatros terrestres. Luego, la conflagración se trasladó al mar y al océano. Y, finalmente, los conflictos alcanzaron la altura de la atmósfera y la estratosfera.

Ha sido cierto, de igual manera, que la extensión de los enfrentamientos armados a nuevos escenarios del planeta ha sido el producto directo de los procesos de desarrollo científico propio de las naciones y, por ende, de sus ejércitos. No hubiera sido posible, posteriormente a la constitución del poder terrestre, que surgiera el marítimo, el aéreo y espacial si la tecnología no lo forjara como posible; es decir, sin que los barcos y submarinos alcanzaran las grandes masas de agua, y las aeronaves y dispositivos espaciales ascendieran a las alturas y a la gravedad cero (Liang y Xiangsui, 1999).

\section{Dimensión terrestre}

El deseo de buscar escenarios donde dirimir los conflictos políticos se convirtió en una cuestión perenne a la guerra, debido, en primera instancia, a que el hombre, al ser un ser terrestre, se sintió más cómodo de ejecutar sus batallas en su medio natural. Como afirma Bellamy, la guerra es un fenómeno adscrito históricamente al escenario terrestre (1990, pp. 7-8). Pero la tecnología, por más primitiva que fuera, fue lo que permitió a los ejércitos empezar a ocupar otros espacios del planeta para extender la práctica bélica. Desde esta perspectiva, es importante tener en cuenta que la tecnología se presenta de dos formas: los desarrollos en materia de movilización y transporte y las armas e implementos para la batalla. A continuación, un breve recuento de algunos aspectos ilustrativos de este proceso de transición.

Es preciso reconocer que el primer propósito constructivo del hombre fue crear una industria armamentística que le permitiera protegerse 
de los ataques de los animales (armas defensivas) o atacarlos (armas ofensivas) para conseguir comida y poderse vestir. Al fabricar sus primeras armas, el hombre no piensa en la guerra, sino en su supervivencia. "La guerra sería el producto paulatino cada vez que el hombre se convirtió en sujeto organizado en unidades políticas y vinieron los conflictos por intereses comunes" (Gutiérrez, 1995, p. 85)

Esquemáticamente, se puede dividir la evolución de las armas terrestres para enfrentar la guerra en cuatro etapas durante la modernidad. La primera, la de las armas de fuego durante la época napoleónica; la segunda, durante la Revolución Industrial hasta la Segunda Guerra Mundial; la tercera, los avances durante la Guerra Fría; y, por último, los adelantos del siglo XXI (Martí, 2006).

Introducir el arma de fuego en la guerra fue una innovación de gran magnitud en el ámbito militar. Su potencia explosiva creó la base para desarrollar artefactos con un poder de destrucción mucho mayor. Sin embargo, las primeras armas eran pesadas, lentas e imprecisas. Estas se podían clasificar en dos tipos: individuales, manejadas por una sola persona, y las grupales, específicamente los cañones (Martí, 2006). Este periodo también marcó el surgimiento de ejércitos profesionales, permanentes. "La introducción de las armas pequeñas, como el mosquete, cambió radicalmente el rostro de la guerra. Por primera vez, los militares tenían un arma portátil, relativamente precisa, fiable y mortal"7 (Mehan, 2014, p. 43).

Así, el arcabuz fue evolucionando hacia el mosquete y, a mediados del siglo xix apareció el fusil Mauser, que disparaba tiro a tiro. Posteriormente, ya en el siglo $\mathrm{xx}$, aparecieron los fusiles con un tipo de cierre más avanzado que permitían tirar también a ráfagas. (Gutiérrez, 1995, p. 87)

Los grandes avances en el conocimiento de la física, la química y las matemáticas que tuvieron lugar en el siglo xviII trajeron consigo en el siguiente siglo una Revolución Industrial gracias a la cual se produce un cambio tecnológico de gran magnitud

7 Traducción del autor. 
en los campos de los materiales, la propulsión, la munición y la electricidad que supondría una mejora sustancial del armamento, que a la postre se traduciría en una nueva forma de guerra. (Martí, 2006, p. 101)

Hacia 1877 el fusil de repetición fabricado en acero se convirtió en el arma estándar de los ejércitos. La nueva munición aumentó su velocidad hasta superar el sonido, creciendo así su poder destructor debido a su mayor energía cinética [...]. En este mismo periodo aparece la ametralladora moderna. (Martí, 2006, p. 116)

Respecto del transporte, al principio de la Primera Guerra Mundial surgió la idea de que un vehículo blindado sobre orugas podía ser capaz de cruzar con impunidad las trincheras enemigas. Si se le equipaba con cañones apropiados, este vehículo podía destrozar las ametralladoras enemigas y abrir camino a un avance general. En el año 1920, las fuerzas terrestres tenían gran cantidad de fuerzas dotadas de fusiles de cerrojo, muchos morteros y artillería más pesada. En 1940, tenían más y mejores vehículos blindados y de un 5 a un $10 \%$ de las tropas eran unidades mecanizadas. (Gutiérrez, 1995, p. 89)

Por otro lado, la Segunda Guerra Mundial vivió un desarrollo nunca visto de máquinas de guerra, en el marco de la Revolución Industrial. Antes de finalizar la contienda, aparecieron en el escenario vestigios de una nueva clase de tecnología destinada a dar un valor superlativo a la acción de los ejércitos en el campo de batalla: los carros de artillería (Lewis, 2002). A manera de contexto, los ejércitos avanzados todavía estaban estructurados, como lo habían sido desde la década de 1940, alrededor de fuerzas blindadas complementadas por tropas de infantería ligera que se mueven en vehículos y camiones.

El mundo vio el surgimiento del Abrams estadounidense, el Challenger II británico, el Leopard II alemán, el Merkava Mk. 4 israelí y, por supuesto, los tanques rusos T-80 y T-90. Todos los tanques modernos tienen torretas estabilizadas, capacidades de visión nocturna, localizadores de rayos láser y equipos de orientación que les permiten luchar en condiciones adversas, en movimiento o en 
la oscuridad, que hubieran obstaculizado los modelos anteriores. Además, la armadura compuesta o reactiva ofrece mucha más protección que en años anteriores, y las armas principales que disparan rondas de uranio empobrecido tienen mucho más poder de penetración (Boot, 2006, p. 14).

Se podría señalar que la informatización y automatización de las cuales se habló en páginas precedentes también impactaron el desarrollo de las capacidades de actuar en tierra. En los Estados Unidos, a partir de 1980, se registró el inicio de proyectos en torno al desarrollo de carros militares sin tripulación (unmanned ground vehicles [UGV]), con las posibilidades de efectuar lanzamiento de armas, reconocimiento, vigilancia y adquisición de objetivos y eliminación de artefactos explosivos (Thong, Howe y Lee, 2012, p. 16).

\section{Dimensión marítima}

En la dimensión marítima, la evolución tecnológica también afectó el progreso del poder naval. Al igual que el combate en tierra, las luchas en los mares y océanos ya se habían institucionalizado a gran escala prácticamente desde las guerras entre el Imperio persa y los griegos. El desarrollo de acorazados y barcos desde el siglo xIx y la puesta en escena de submarinos y portaaviones en el siglo xx permitió el desarrollo de, como se podría expresar conforme a las ideas del almirante Thayer Mahan, una verdadera estrategia geopolítica de control de las vías de transporte comercial marítimo y un mecanismo de disuasión frente a un ataque a la nación (Mahan, 1911).

Una flota de buques podría controlar los mares y garantizar a una nación una posición como una potencia marítima. La fuerza naval se midió en el número de buques "líder" o de clase líder. Con esta magnitud de barcos y tipos de barcos, la dependencia tecnológica de la guerra en esta dimensión no se hizo esperar y, así mismo, se derivaron nuevas tácticas de la guerra naval (Fleming, 1993, p. 1). Con esa fuerza, surgió la capacidad de influir en el estatus económico y político de la nación. Además, si esa nación estaba involucrada en algún tipo de operación de tierra ofensiva o defensiva, las fuerzas navales se emplearían a menudo. Su empleo tendría una influencia decisiva en el resultado. Una vez que 
ese compromiso fue activamente perseguido, la Marina se convirtió en un elemento clave en la estructura defensiva de la nación al mantener una postura en general ofensiva (pp. 3-4).

El avance tecnológico trajo rápidamente a las flotas navales de los Estados buques más resistentes en combate. La propulsión de vapor permitió a los buques decidir sus destinos con libertad, ya que el viento no era un factor determinante en la batalla. El trabajo con el acero, propio de la industrialización, permitió generar blindajes a los barcos de guerra, así como armamento con capacidad explosiva. Esto dio origen al acorazado moderno a finales de 1800; recordemos los buques pre-Dreadnought y Dreadnought. (pp. 6-7)

Otro ejemplo de la expansión y el avance tecnológico de la guerra en la dimensión marítima fue la invención del torpedo autopropulsado en Gran Bretaña en 1866. Este primer modelo era un bote de hélice que portaba una carga explosiva, en el cual un mecanismo de relojería accionaba la hélice y uno de percusión hacía funcionar la carga. Alcanzaba un blanco a $1600 \mathrm{~m}$ a una velocidad de 40 nudos. En la Primera Guerra Mundial, los alemanes adaptaron el torpedo al submarino, lo que causó el $60 \%$ de las pérdidas aliadas en navíos mercantes (Gutiérrez, 1995, p. 88).

Nada revolucionó tanto la guerra naval como los submarinos, los cuales se pusieron a prueba por primera vez durante la guerra civil norteamericana. En 1864, el submarino confederado css H. L. Hunley hundió el uss Housatonic en Carolina del Sur. Desde entonces, los submarinos han tomado muchas formas diferentes y se han utilizado en diferentes guerras (Khan, 2015). Durante la primera mitad del siglo XIX, se diseñaron varios tipos de submarinos, entre ellos, el Ictineo de Monturiol, movido por máquinas de vapor. En 1903, los periscopios proporcionaron a los submarinos el medio por el cual podían apuntar los torpedos hacia sus blancos mientras permanecían sumergidos (Gutiérrez, 1995, p. 89).

Ya para la década de 1960, algunos submarinos estaban equipados con misiles balísticos intercontinentales, pero su orientación 
era tan imprecisa que no tenía sentido equiparlos con ojivas convencionales. Los misiles balísticos se convirtieron en un pilar de la disuasión nuclear. El desarrollo de misiles de crucero precisos que comenzaron en la década de 1970 permitió que los submarinos y los combatientes superficiales golpearan los blancos de la tierra a centenares de millas con la artillería convencional ${ }^{8}$. (Boot, 2006, p. 16)

En la década de 1960, la marina de guerra de los Estados Unidos desarrolló un vehículo submarino controlado por cable para operaciones de rescate y recuperación de objetos desde el fondo del océano. En la década de 1970, los vehículos subacuáticos autónomos tempranos fueron desarrollados por los Estados Unidos y la antigua Unión Soviética. (Thong, Howe y Lee, 2012, p. 17)

Los misiles Tomahawk son otro ejemplo de cómo la guerra instó a las naciones a proponer nuevas tecnologías. Son misiles de crucero de largo alcance que tienen la capacidad de volar a baja altitud a una velocidad increíble. Pueden ser utilizados por los buques para golpear cualquier área de superficie. Las posibilidades de una falta son casi inexistentes, porque estos misiles vienen con un receptor GPS (global positioning system 'sistema de posicionamiento global') que se utiliza para localizar la ubicación del objetivo con mayor precisión (Khan, 2015).

\section{Dimensión aérea}

Se harán ahora algunas consideraciones acerca de la dimensión del aire. Hasta la Primera Guerra Mundial, los líderes militares habían concebido el avión principalmente como una herramienta de reconocimiento y de localización de artillería. A finales de 1918, sin embargo, el avión ya estaba realizando otras misiones, que incluyen la superioridad aérea, el bombardeo estratégico, la interdicción, el apoyo aéreo cercano y el transporte aéreo. La aviación siguió evolucionando después de la

8 Traducción del autor. 
guerra, como lo demuestra el aumento de los rangos, las altitudes y las velocidades de los aviones. Estas capacidades crecientes permitieron vuelos transcontinentales y transoceánicos, así como el servicio de aerolíneas y el correo aéreo. El Servicio Aéreo del Ejército de los Estados Unidos (U.S. Army Air Service) realizó el primer vuelo alrededor del mundo en 1924 y demostró el alcance global de la potencia aérea. Los monoplanos de metal con cabinas cerradas y el tren de aterrizaje retráctil reemplazaron los biplanos de piel abierta y de cabina abierta con ruedas fijas. El Cuerpo Aéreo del Ejército, establecido en 1926, desarrolló grandes bombarderos de largo alcance y una doctrina para su uso (Haulman, 2003).

Es importante conocer la evolución de los avances tecnológicos que han permitido a los actores, principalmente políticos, comerciales y militares, gobernar el cielo. El primer Piper J-3 Cub salió a la venta en 1938. Con la guerra que amenazaba en Europa, el pequeño Cub se convirtió en un entrenador principal para el Programa de Capacitación de Pilotos Civiles. Para el final de la Segunda Guerra Mundial, el 80 \% de todos los pilotos militares de los Estados Unidos recibieron su entrenamiento primario en un J-3. La construcción simple del Piper, su bajo costo y su manejo dócil lo convirtieron en uno de los aviones más populares de todos los tiempos (Clarke, 2015).

Poco antes de la Segunda Guerra Mundial, los científicos e ingenieros británicos descubrieron cómo utilizar la reflexión de las ondas electromagnéticas para localizar aviones a una distancia que permitiera a pilotos de caza interceptarlos. La serie de estaciones radar que montaron los ingleses, atentas a los vuelos procedentes del continente, se mostraron determinantes en la señalización de aviones de bombardeo alemanes durante la batalla de Inglaterra. El radar siguió desarrollándose muy rápidamente durante la guerra y encontró nuevos usos en la navegación de los aviones, el tiro y la técnica aeroespacial (Gutiérrez, 1995, p. 90).

El Supermarine Spitfire fue el único avión británico en producción continua durante toda la Segunda Guerra Mundial. Se convirtió en la espina dorsal del Royal Air Force Fighter Command y fue más conocido por derrotar a la Luftwaffe alemana durante la batalla de Inglaterra. Fue diseñado para llegar a velocidades más altas que muchos otros combatientes (Clarke, 2015). 
Aunque los problemas del motor retrasaron su estado operacional con la Luftwaffe alemana, en 1942 el Schwalbe (Swallow) se convirtió en el primer avión de combate jet del mundo. Su efectividad se vio obstaculizada por problemas de fiabilidad del motor y ataques aliados a los suministros de combustible alemanes. El avión también tenía una vida útil limitada de producción, pero su velocidad y maniobrabilidad eran incomparables en ese momento y su diseño todavía se estudiaría y se aplicaría a futuros aviones de combate como el F-86 Sabre. El Bell X-1 es un avión de investigación supersónico famoso por ser el primer avión tripulado capaz de romper la barrera del sonido en 1947. Fue el primero en combinar tecnologías de aviones y cohetes. Estos aviones experimentales se usaron para probar sistemas avanzados y aerodinámicos, y las lecciones aprendidas impulsarían a los Estados Unidos en su carrera en el espacio. Además, los datos de vuelo supersónicos recogidos de pruebas X-1 resultaron invaluables para aquellos que diseñaron futuros aviones de combate estadounidenses (Clarke, 2015).

La llegada de cazabombarderos y de aviones espías de la Guerra Fría ha configurado el poder aéreo contemporáneo (Douhet, 2009). Pero también el ejemplo más reciente del avance en la tecnología tiene que ser los aviones furtivos. Los radares están ahora activos en casi todos los países, lo que significa que nada pasa desapercibido. Sin embargo, es una situación completamente diferente si existen aviones furtivos que se esconden de los radares en cierta medida. La tecnología Stealth hace que sea realmente difícil para los enemigos detectar el avión, lo que quiere decir que van a necesitar tiempo para encontrarlo, seguirlo y luego defenderse contra él. Los Estados Unidos tienen tres tipos de aviones sigilosos: F-35 Lightning, F-22 Raptor y B-2 Spirit (Khan, 2015).

El primer uso de la tecnología de aire no tripulada se remonta a la Primera Guerra Mundial, cuando los aviones no tripulados controlados por radio fueron utilizados como "bombas volantes" por los Estados Unidos. A partir de entonces, tales vehículos aéreos no tripulados fueron usados como señuelos. Luego, se utilizaron para fines de reconocimiento en la década de 1960 y, para la década de 1970, los Estados Unidos experimentaron con ellos para propósitos 
de combate activo. Sin embargo, no fue hasta la década de 1990 cuando los vehículos aéreos de combate no tripulados fueron desarrollados y utilizados en operaciones, con el avance de enlaces de comunicación más confiables (Thong, Howe y Lee, 2012, p. 16).

El Predator fue el primer dron militar (aunque el término más preciso sería “vehículo aéreo no tripulado"). Se hizo famoso por su papel en la lucha contra los talibanes en Afganistán. El Predator puede ser pilotado remotamente para volar sobre un curso de 400 millas náuticas, rodear su objetivo por hasta 14 horas y volver a la base. El uso extensivo del Predator, no solo para recopilar información, sino también para disparar misiles dirigidos por láser Hellfire, marcó el comienzo de la era moderna de la guerra extensa de los aviones no tripulados por el Ejército estadounidense (Clarke, 2015).

\section{Dimensión espacial}

A manera de cierre, es un deber tener en cuenta el paso más grande que ha dado el ser humano para buscar nuevas dimensiones dónde realizar la guerra: la dimensión del poder espacial. Esta, al igual que su consideración como un poder estatal (militar), ha sido una cuestión de exclusividad. Aunque en la actualidad existen países que por sus Рів y su capacidad de deuda pública no pueden invertir demasiados recursos en sus presupuestos de defensa, de una manera u otra, han logrado desarrollar o adquirir capacidades para proteger o asumir una conducta bélica en la dimensión terrestre, marítima y aérea. No obstante, tanto alcanzar la dimensión espacial, la estratosfera del planeta y la Luna, como constituir un programa científico y tecnológico que permitiera desempeñar misiones y tácticas para cumplir a su vez estrategias, han sido un privilegio de pocos Estados nación. Es probable que en la actualidad en gran parte de los países del mundo se estén desarrollando artefactos que permitan a sus usuarios públicos o privados desarrollar nuevos procesos, pero, si se habla desde el punto de vista de la seguridad nacional, y de la guerra, todo se traduce en cuál es el número de países que en realidad tienen la capacidad de colocar dichos recursos en esta dimensión.

En otras palabras, y según Fitzgerald (2001), 
el poder espacial se aplica en el caso específico de los actores del sistema internacional que lograron, y podrían hacerlo en el futuro, desarrollar la tecnología para rebasar la atmósfera terrestre mediante cohetes de propulsión o transbordadores, y poseer el control del espectro electromagnético del planeta mediante los satélites de carácter geopolítico, es decir, artefactos encaminados al aumento del poder político-militar, y no simplemente comercial ${ }^{9}$. (citado por Haulman, 2003, p. 11)

Debe reconocerse, como lo comenta Millán, que el reto más grande que se ha presentado para consolidar la conquista del espacio exterior ha sido garantizar en primera instancia un acceso seguro a este. Situar una astronave en órbita demanda un mecanismo - motor- de propulsión que genere una velocidad mínima de 28440 km/h (2000, p. 209).

Se podría señalar entonces que el poder espacial presentó tres momentos de cercanía con la guerra. Primero, en lo que podría denominarse las raíces del poder espacial, momento que se caracteriza por el desarrollo de la bomba Aggregat 4 (A4), o más conocida como la bomba V2 en Alemania durante la Segunda Guerra Mundial (National Aeronautics and Space Administration [NASA], s. f.). Segundo, las décadas de 1950 a 1970, cuando alcanzar el espacio y poner el primer hombre en la Luna se convirtieron en una cuestión de orgullo nacional, la cuestión más representativa de la guerra político-cultural de aquel enfrentamiento capitalismo versus comunismo. Y tercero, entre las décadas de 1980 y 1990, momento en el que las capacidades espaciales se enfocan desde una perspectiva político-militar en garantizar aspectos de la seguridad nacional, como evitar ataques de misiles balísticos intercontinentales, o desde plataformas submarinas que lograran acercarse al rango de distancia requerido para lanzar artefactos nucleares (Grauer, 2013).

Wernher von Braun fue reconocido durante la Segunda Guerra Mundial por haber construido la bomba V2 (A4) para el Ejército alemán. Aunque técnicamente el arma no estuvo dispuesta en su capacidad máxima para ser empleada en la guerra, en especial por los

9 Traducción del autor. 
bombardeos aliados sobre la fábrica, se reconoció el ingenio de Von Braun para construir un misil (cohete) con capacidad de alcanzar objetivos a $200 \mathrm{~km}$ de distancia, y se calculó que alcanzó altitudes que oscilaban entre los 50 y $55 \mathrm{~km}$ de altura; además de esto, tenía una capacidad destructiva de una tonelada de trinitrotolueno (TNT). Al finalizar la guerra en 1945, cargas de V2 y algunos de sus ingenieros fueron trasladados a los Estados Unidos. Uno de los mejores resultados de la Operación Paperclip fue el traslado de Von Braun a Norteamérica, pues su conocimiento fue determinante para todo el desarrollo de misiles, como el Redstone, en la División de Operaciones de Desarrollo de la Agencia de Misiles del Ejército de los Estados Unidos, así como para los primeros cohetes de la NASA (National Aeronautics and Space Administration 'Administración Nacional de la Aeronáutica y del Espacio'), como fue el Júpiter C (Haulman, 2003, p. 71).

Respecto de la segunda etapa que se planteó, se podría señalar que la antigua Unión Soviética inició ganando la carrera espacial de la Guerra Fría al poner en órbita el satélite Sputnik I y el Sputnik II, en el cual además viajó Laika, el primer ser vivo que el hombre puso en el espacio (NASA, s. f.).

Durante 1958, la Fuerza Aérea de los Estados Unidos (United States Air Force [USAF]) puso en órbita el primer satélite artificial de comunicaciones, artefacto que un día después de posicionado en órbita logró transmitir un mensaje de Navidad del presidente Dwight D. Eisenhower, el primer comunicado de una persona transmitido desde el espacio (Haulman, 2003, p. 84). En 1960, la NASA inició el programa Mercurio, el cual, a diferencia de la antigua Unión Soviética, no solo buscaba llevar dispositivos y animales al espacio, sino a los primeros seres humanos (p. 85). En respuesta a lo anterior, Yuri Gagarin, un cosmonauta soviético, fue el primero en orbitar la Tierra; aunque lo prosiguiera el astronauta Alan Shepard unos meses después gracias a las metas alcanzadas por Mercurio. Al final de 1950, los Estados Unidos y la antigua Unión Soviética transportaron satélites al espacio, con lo cual transformaron la comunicación, la navegación, la inteligencia y la exploración.

Es evidente que uno de los grandes paradigmas de la guerra en el espacio se dio en 1969 cuando, desde el Centro Espacial Kennedy en 
Florida, se coordinó toda la operación para permitir que, de un módulo lunar de la NASA, la tripulación de Apolo 11, Neil Armstrong, Michael Collins y Edwin E. Aldrin fueran los primeros terrícolas en caminar sobre el satélite natural de la Tierra (Haulman, 2003, p. 107).

En una última etapa, el programa militar estadounidense lanzado por el presidente Ronald Reagan en 1983 con la denominación de Iniciativa de Defensa Estratégica fue rotulado por los medios de comunicación como "guerra de las galaxias" (Fitzgerlad, 2001). El objeto del programa consistió en disponer de capacidades balísticas, ubicadas desde baterías terrestres y plataformas espaciales, para destruir los misiles nucleares dirigidos a blancos estadounidenses. El procedimiento previsto para esto consistía en detectar cuanto antes el disparo de los misiles, seguir sus trayectorias y destruir las cabezas nucleares a suficiente altura para que sus efectos no afectasen a los blancos. Todo esto debería conseguirse en pocos minutos, los veinte más o menos que medían entre los momentos del disparo del misil y del impacto de la cabeza de guerra en el blanco (Millán, 2000, p. 219). El espacio es también objeto de una utilización muy importante en programas militares para muy diversas misiones. Su uso ha sido fundamental en la previsión y el control de crisis y en operaciones como la guerra del Golfo Pérsico de 1990 (p. 218).

Actualmente, una cantidad creciente de vigilancia, comunicaciones y trabajo de inteligencia está siendo realizada por aviones no tripulados y satélites. En 2001, los Estados Unidos tenían aproximadamente cien satélites militares en órbita. Este país gasta más de USD 15000 millones al año en el espacio militar, por lo cual ha logrado que muchos de sus satélites de vigilancia puedan visualizar un objetivo de seis pulgadas de tamaño desde 150 millas de altura. Una nueva generación de satélites utiliza tecnología furtiva para que otros países no puedan rastrear sus movimientos y así saber cuándo esconder el equipo de los ojos estadounidenses (Boot, 2006, p. 21).

Durante la última década del siglo xx, la tripulación del transbordador espacial Endeavour completó la primera misión de montaje de la Estación Espacial Internacional, que incluyó la entrega y el acoplamiento de Unity, el primer módulo de los Estados Unidos, al módulo de Zarya, lanzado por Rusia. Los miembros de la tripulación también 
lanzaron la USAF MightySat I para evaluar materiales compuestos, células solares avanzadas y otras tecnologías. Desde Cabo Cañaveral (Florida), un cohete Delta II lanzó Stardust, la primera nave espacial diseñada para interceptar un cometa y devolver muestras a la Tierra. La NASA programó que Stardust se reuniera con el Cometa Wild-2 el 2 de enero de 2004. China lanzó, supervisó, controló y desembarcó Shenzhou, su primera nave espacial no tripulada (Haulman, 2003).

Antes de concluir este capítulo, es valioso reconocer que a lo largo de estas páginas han faltado innumerables hechos, armas, operaciones, procesos de desarrollo científico, características y causas de la guerra que sin duda han sido determinantes en la historia de este fenómeno. No obstante, es importante recordarle al lector que la intención no era hacer un barrido específico de toda la evolución de la tecnología en la historia de la guerra o bien de todos los aspectos de la naturaleza que pueden definir esta práctica humana. La intención era ofrecer algunos datos para que el lector pudiera hacerse un contexto básico de unos elementos que son destacables en lo que, a juicio del autor, pueden dar explicación y lucidez frente al fenómeno de la ciberguerra, el cual para algunos es novedoso o confuso.

Se ha discutido sobre temas que han sido conceptualizados por muchos analistas para llegar a la noción o la idea de qué es lo que significa cuando dos actores políticos entran en un estado de guerra, y qué debe existir en este escenario para suponer que lo que está pasando allí puede recibir o amerita la categoría de guerra. De ahí la importancia de haber analizado los primeros elementos constitutivos de la guerra, que fueron planteados en este capítulo. Y, en un segundo punto, reconocer, como se verá de manera mucho más clara en el siguiente capítulo, la guerra y el desarrollo científico y tecnológico a lo largo de la historia en las dimensiones donde se llevó a cabo.

Para dar un enfoque mucho más claro en torno a estas capacidades tecnológicas, se mostró cómo influyeron en el fenómeno de la guerra, también dando a entender que la invención humana ha permitido en primera instancia que el hombre afiance las prácticas bélicas en su espacio connatural, que es la tierra. Además, se presentan nuevos escenarios, los cuales dominados por las capacidades humanas permitieron simultáneamente reconocer el desarrollo de artefactos que 
pudieran gobernar dimensiones que parecían imposibles de alcanzar. Asimismo, cómo estos preconceptos fueron permeados por unas capacidades tecnológicas para permitir enfrentamientos en un entorno en el cual no existe un desempeño de manera connatural como sí lo pueden hacer otras especies.

Cuando se piensa en el mar, el aire y en el espacio como dimensiones que se alejan de la naturaleza y las características de hábitat propias del hombre, ha existido un aprendizaje sobre la generación de actividades como seres humanos en los espacios marítimos y oceánicos, aéreos y espaciales, si bien siempre se ha hallado la imposibilidad de vivir en dichos espacios de manera permanente. Así es que, en últimas, con mayor razón, el desarrollo científico y tecnológico permitió expandir las fronteras de la guerra para llevarla al mar, al aire y, aún más, al espacio. Y está, por supuesto, la otra idea que se quería aclarar a partir de este capítulo, pues, en últimas, y ya para concentrarse verdaderamente en competencia, esta evolución tecnológica que ha sobrepasado los límites permitió una perspectiva específica y diferente que se consolidará en una nueva dimensión para la guerra, que es de lo que trata el siguiente capítulo. 
